\title{
Assessment of a multimodal analgesia protocol in goats undergoing claw amputation

\author{
Usama Fekry ${ }^{1}$, Awad Rizk $^{2}$, Esam Mosbah ${ }^{2}$ and Adel Zaghloul ${ }^{2}$ \\ ${ }^{1}$ Veterinarin, Veterinary Sector, Dakahlia, Egypt \\ ${ }^{2}$ Department of Surgery, Anesthesiology and Radiology, Faculty of Veterinary Medicine, Mansoura University, 35516 Mansoura, Egypt
}

\section{ARTICLE HISTORY}

\section{Received: 24.09 .2019}

Revised: 26.10 .2019

Accepted: 28.10 .2019

Address correspondence Awad Rizk; Tel: +201099513339;

E-mail: awad_surgery@yahoo.com

\section{ABSTRACT}

Objective: To evaluate the anti-nociceptive, sedative and behavioral effect of multimodal analgesic drugs in goat's undergoing unilateral claw amputation.

Design: A randomized experimental design.

Animals: Sixteen goats were randomly assigned into 4 groups (4 each).

Procedures: Animals used in this study were randomly assigned into 4 groups, four animals for each group. All goats were anaesthetized by intravenous regional anesthesia (IVRA) of the right forelimb cephalic vein with $2 \%$ lidocaine in a dose of $3 \mathrm{mg} / \mathrm{kg}$ body weight (BW) before surgery. Administration of carprofen $(50 \mathrm{mg} / \mathrm{ml}$ ) at a dose $0.1 \mathrm{mg} / \mathrm{kg} \mathrm{BW}$ took place before surgery on day 0 and was then repeated once daily for another 3 consecutive days postoperatively in groups Lidocaine (L), carprofen_lidocaine (CL), carprofen - romifidine lidocaine $(C R L)$, and carprofen - romifidine - butorphenol - lidocaine (CRBL). Heart rate $(H R)$, respiratory rate (RR), rectal temperature (RT), serum IL-6 level, sedation score, and lameness score were determined at preset time intervals and the animals' behavior was recorded.

Results: The mean average of HR, RR and RT in IVRA treated animals was significantly increased until the end of surgery followed by gradual decrease up to 90 min post-surgery. In CRL and CRBL it showed a significant decrease compared to controls. There was a significant decrease in lameness score in CRL and CRBL treated animals from 30 min until 2 hours and for 3 days post-surgery. After injection of romifidine it showed a significant decrease of IL- 6 levels compared to lidocaine and CL treated animals.

Conclusion and clinical relevance: It was concluded that, the use of romifidine and buterphanol in the analgesic protocol during claw amputation in goats, alleviate pain response and potentiate the analgesic effects of IVRA. Addition of carprofen to the analgesic protocol can be used for long- term treatment of orthopedic pain in goats without complications.

Keywords: IVRA, Carprofen, Goats, Claw amputation, Romifidine, Buterphanol

\section{INTRODUCTION}

Lameness in dairy goats has been identified as a common welfare problem [1]; however, the diseases causing lameness are less well described than in cattle [2,3] and sheep [4]. Generally, goat lameness problems are described as similar to those found in sheep. However, interdigital dermatitis [4, 5], foot rot[6], heel horn erosion [7], white line disease, foreign body penetrations [8] and overgrown feet [1] have all been recorded in goats.

As lameness can cause significant losses in both welfare and production it is an important topic for all livestock. Goats are generally underrepresented in scientific papers, so we often refer to information from other species or our own experience when dealing with a lame goat, whether it is an individual pet goat or a herd problem. In recent years, however, more goat specific knowledge on lameness has surfaced. It seems goat lameness is as the animal itself, seemingly similar to other species but with a wholly different character[9].
For optimal pain relief it has been recommended to combine sedation, local anesthesia, opioids and non-steroidal anti-inflammatory drugs (NSAID)[10]. The NSAID, carprofen, has recently been shown to have some efficacy in alleviating the adverse behavioral responses of lambs to mulesing[11, 12]. Intravenous regional anesthesia (IVRA) is the technique of choice for surgery of the distal bovine limb[13, 14], as it has a rapid onset, is easy to perform and more reliable than local nerve blocks [15]. IVRA is a type of local anesthesia and during multimodal pain; management may be supplemented by sedatives or the use of NSAIDs. In small ruminants, IVRA is less easily applied than in bovine, obviously because of small size[15]. IVRA is indicated in goats for the removal of interdigital cysts, interdigital papillomata, surgical treatment of wounds of the foot, lower leg amputation, and any other conditions of the distal limb requiring short surgical procedure[15].

To our knowledge, no available data about the use of carprofen for postoperative pain control during orthopedic intervention in goats. Therefore, the aim of the present study 
is to evaluate the effect of multimodal analgesic protocols on cardiopulmonary and behavioral changes as well as on lameness and sedation scores and on the level of serum IL- 6 and lactate in goats during unilateral claw amputation.

\section{MATERIAL AND METHODS}

\subsection{Animals and housing}

Sixteen apparently healthy goats at 8-12 months of age and average body weight of $15-20 \mathrm{~kg}$ were used. All animals were free from concurrent diseases. All animals were kept in individual free stalls on straw bedding and were fed a diet based on hay and concentrates. No medications were given within one week before the experiment. Food was withheld for 24 hours and water was for 6 hours before surgery. This study was applied in Mansoura Veterinary Teaching Hospital of Mansoura University, Mansoura, Egypt.

\subsection{Experimental design}

Animals used in this study were randomly assigned into (4) groups, four animals for each group. Goats were anaesthetized by IVRA in right cephalic vein (Fig 1) with lidocaine hydrochloride (Debocaine 2\%, Al- Debeiky pharmaceutical industrial co, Egypt) $2 \%$ in a dose of $5 \mathrm{mg} / \mathrm{kg}$ before applying surgery. Initial administration of carprofen (Carprofen, $50 \mathrm{mg} / \mathrm{ml}$, Adwia co, Egypt) at a dose $0.1 \mathrm{mg} / \mathrm{kg}$ B.Wt took place before surgery on day 0 and was then repeated once daily for another 3 consecutive days postsurgery in all groups .

Group 1: Lidocaine (L):

Animals in this group were fixed on lateral recumbancy with the leg to be anaesthetized lower most for application of IVRA

\section{Group2: Carprofen - Lidocaine (CL).}

Animals in this group were intravenously (IV) received carprofen then after $15 \mathrm{mins}$, the animals were cast on lateral recumbancy with the leg to be anaesthetized lower most for application of IVRA.

Group3: Carprofen -Romfidine - Lidocaine (CRL).

Animals in this group were received carprofen IV then after $15 \mathrm{mins}$, romfidine (Sedivet $10 \mathrm{mg} / \mathrm{ml}$ bohehringer ingelheim, Burlington, Germany) at a dose $0.5 \mathrm{ml} / \mathrm{kg}$ then lateral recumbancy for application of (IVRA) with lidocaine hydrochloride $2 \%$.

Group 4: Carprofen - Buterphenol - Romfidine - Lidocaine (CRBL).

Bucks in this group were received carprofen IV then after $15 \mathrm{mins}$, romfidine at adose $0.5 \mathrm{ml} / \mathrm{kg}$ then buterphenol at adose $0.2 \mathrm{ml} / \mathrm{kg}$ then restrained into lateral recumbancy for application (IVRA) with lidocaine hydrochloride $2 \%$

\subsection{Monitoring}

Cardio-respiratory parameters: Heart rate (HR) was recorded by counting the heart beats per min by auscultation. Respiratory rate (RR) by counting thoracic excursions per min and rectal temperature (RT) was recorded using a digital thermometer.

Serum IL-6 and lactate level: Blood samples were centrifuged at $3000 \mathrm{rpm}$ for 10 minutes, and the separated sera were stored at $-80{ }^{\circ} \mathrm{C}$ until assay. Interleukin- 6 levels by using goat IL-6 ELISA kit (Cusabio Biotech Co., LTD, China) and Lactate (Lactate, BioSystems S.A., Spain).

Behavioral monitoring: Behavioral signs were recorded using video camera according to[16, 17] (Table 1).

Sedation score: was documented by a score system modified after [18] (Table 2).

Lameness score: was recorded according to[1] (Table 3).

\subsection{Surgical approach: Digit amputation}

For application of IVRA, the animal was cast on lateral recumbency with the leg to be anaesthetized lowermost. A rubber tourniquet (Esmarch rubber bandage) about $3 \mathrm{~cm}$ wide and $20 \mathrm{~cm}$ long was securely applied above the elbow joint. The medial aspect of the forearm was thoroughly shaved and disinfected. The cephalic vein was located in the middle third of the forearm where it crossed to the anterior part of the limb from the medial aspect (Figure 1A). A 3-4 ml lidocaine hydrochloride $2 \%$ was injected (Figure $1 B$ ). The skin is incised in an upside-down $T$ pattern laterally: the longitudinal incision runs abaxially of the distal third of P1 to the coronary band, and the horizontal incision runs in the coronary band around the claw (Figure 2A). The skin flaps are undermined and reflected forwards and backwards, respectively, and proximally, and the claw is removed by disarticulating the pastern joint (Figure 2B, C). The cartilage of the distal joint surface of proximal phalanx is thoroughly curetted, and, if necessary, the interdigital fat pads are reduced to enable skin closure (Figure 2D). The skin flaps are pulled over the stump and sutures closed using simple interrupted suture pattern by silk (Figure 2E). This technique is modified according to the method described previously[19] in cattle.

\subsection{Post-operative care}

All goats received IM of cefotax (cefotaxime $50 \mathrm{mg} / \mathrm{kg} /$ twice daily, EIPICO, Egypt) for 5 days. Bandage was changed once a week and Local antiseptic dressing with dilute liquid povidone iodine was done. Skin suture was removed 10 day after healing (Figure 2F).

\subsection{Time table}


Baseline values were determined 15 min before drug administration. HR, RR and RT were measured after administration of anesthetic agent, during surgery, end of surgery and then at 15, 30, 60, 90 and 120 minutes postoperatively and at 1, 2 and 3 days postoperatively. Blood samples were drawn for cytokines and metabolic parameters measurement at regular preset time interval.

\subsection{Statistical analysis}

Data were analyzed using one-way ANOVA showed significant differences among groups. Analysis was performed with the software SPSS version 16.0 (SPSS Inc, USA). To determine which groups are different, the data were analyzed by Dunnett's test was used to compare the experimental groups. A probability value less than $0.05(\mathrm{P}<$ 0.05 ) was considered significant.

\section{RESULTS}

\subsection{Results of cardio-respiratory parameters}

The mean average of $\mathrm{HR}$ in IVRA treated animals was significantly increased $(P<0.05)$ until the end of surgery followed by gradual decrease up to 90 minutes post-surgery (Table 4).

In $\mathrm{CL}$ treated animals the mean value of $\mathrm{HR}$ was increased significantly followed by a gradual decreased until the end of surgery and increased up to 90 minutes postsurgery. There was a significant decrease in $\mathrm{HR}(\mathrm{p}<0.05)$ after administration of romifidine compared to first and second group and the lowest value which recorded at the end of surgery was (73.75 \pm 3.5 ; Table 4$)$.

A significant decrease in HR $(p<0.05)$ was shown after administration of buterphenol and romifidine compared to other treated group and the decreasing in HR lasted till 120 minutes post-surgery the lowest value recorded at the end of surgery was $57.52 \pm 2.21$ (Table 4 ).

The mean average of RR was significantly decreased $(p<0.05)$ until 30 minutes post-surgery compared to other groups and based line value the lowest value at the end of surgery was (15.25 \pm 2.38$)$ Romifidine and buterphenol combinations decreased RR significantly up to 30 minutes post-surgery followed by a gradual increase (Table 4 ).

There was no significant change in RT in IVRA treated animals. There was no significant change in RT in $\mathrm{CL}$ treated animals compared to base line value. There was no significant change in CRL treated animals compared with base line. There was no significant change in CRBL treated animals compared with base line (Table 4).

There was a significant increase in RT of CRBL treated animals after injection of anesthetics compared to other group. There was a significant decrease after injection of CRBL treated goats compared to first group at $120 \mathrm{~min}$ postsurgery $(37.95 \pm 1.07,38.87 \pm 1.06,38.75 \pm 0.23)$ respectively (Table 4 ).

\subsection{Results of behavioral changes}

The frequency of struggling and vocalization was higher in lidocaine treated animals compared to other group (Table 5).

Administration of romifidine results in a decreased vocalization and struggling for two hours compared to the first and second group Romifidine-Buterphenol combinataion decreased vocalization and struggling 2 hours compared to first and second group (Table 5).

Ear flaking and head shaking was significantly $(P<0.05))$ higher in IVRA and $\mathrm{CL}$ treated animals compared to other group (Table 5).

\subsection{Sedation score results}

There was no sedation in IVRA and $\mathrm{CL}$ treated animals. After injection of romifidine there was a moderate sedation lasted till 120 min post-surgery. Addition of buterphenol to the combination showed a marked sedation which also lasted till 120 min post-surgery (Table 6).

\subsection{Results of lameness score}

There was a significant increase in lamness garde in IVRA treated animals; which subsequently significantly decreased in CRL and CRBL treated animals from 30 min post-surgery until 2 hours and for 3 days post operatively. Both romifidine and buterphenol administration has sedative and analgesic effect in goats undergoing claw amputation (Table 7).

\subsection{Results of cytokines (IL-6)}

The mean average value of IL- 6 Levels remained significantly $(P<0.05)$ lower in romifidine treated animals compared to other treated groups (Table 8).

The mean average of IL6 in IVRA treated animals was significantly increased $(P<0.05)$ during surgery, 60 and 120 $\min$ post-surgery $(7.26 \pm 0.57,8.71 \pm 1.33$ and $7.1 \pm 1.53$ respectively) compared to base line values $(3.57 \pm 0.49)$ (Table 8)

The result of IL-6 in CL treated animals was significantly increased compared to baseline values. There was a significant decreased during surgery in CRBL treated animals compared to baseline value (Table 8).. After injection of romifidine it showed a significant decreased of IL-6 $(P<0.05)$ compared to lidocaine treated animals and $\mathrm{CL}$ treated animals. IL-6 levels remained significantly $(p<0.05)$ lower in CRBL treated animals compared with other groups (Table 8).

\subsection{Results of lactate}


The mean serum lactate level showed a significant increase in Lidocaine treated animals at end of surgery and 30 min P.O (57.9 \pm 24.5 and $50.95 \pm 8.4$ ) respectively compared with base line. There was a significant increase in $\mathrm{CL}$ at $30 \mathrm{~min}$

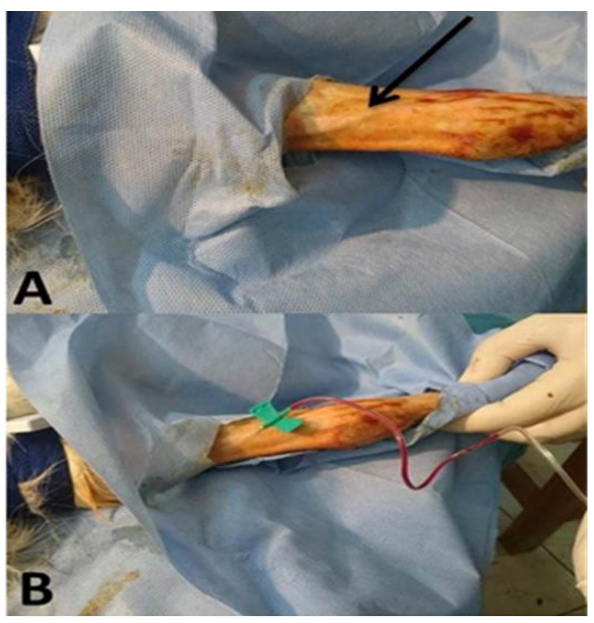

Figure 1. Shows the cephalic vein at the middle third of the forearm in goat (A), butterfly catheter application in cephalic vein (B).

Table 1. Description of behavioral signs recorded during the experiment according to numeric rating scale (NRS).

\begin{tabular}{|c|c|}
\hline Behavior & Description \\
\hline $\begin{array}{l}\text { Struggles } \\
\text { (score) }\end{array}$ & $\begin{array}{l}\text { Slight or vigorous movement of legs, and } \\
\text { attempt to escape } \\
0=\text { no struggling } \quad 1=\text { struggling with hind limbs } \\
2=\text { struggling with hind limbs and/or front limbs } \\
3=\text { struggling with the whole body }\end{array}$ \\
\hline $\begin{array}{l}\text { Vocalization } \\
\text { (n/10 } \mathrm{min})\end{array}$ & $\begin{array}{l}\text { Emission of bleats with open or closed mouth } \\
0=\text { none } \quad 1=\text { once } \quad 2=\text { several times }\end{array}$ \\
\hline $\begin{array}{l}\text { Ear flicking } \\
\text { (n/10 min) }\end{array}$ & $\begin{array}{l}\text { Vigorous movement of one or both of the ears } \\
\text { independent of a head shaking } \\
0=\text { none } \quad 1=\text { once } \quad 2=\text { several times }\end{array}$ \\
\hline $\begin{array}{l}\text { Head shaking } \\
\text { ( } \mathrm{n} / 10 \mathrm{~min})\end{array}$ & $\begin{array}{l}\text { All exaggerated movements of the head without } \\
\text { any discernible reason } \\
\begin{array}{ll}0=\text { none } \quad 1=\text { once } \quad 2=\text { several times }\end{array}\end{array}$ \\
\hline
\end{tabular}

p.o $(44.1 \pm 0.45)$ compared to base line. There was a significant decreased in serum lactate in CRL and CRBL treated animals from end of surgery till $120 \mathrm{~min}$ P.O. (Table 9).

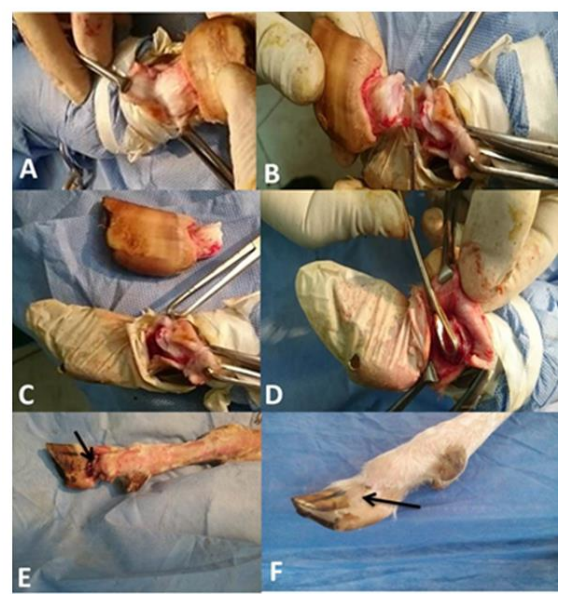

Figure2. Shows a $T$ shaped skin incision above the coronary band (A), the claw is removed by disarticulating the pastern joint $(B, C)$, the cartilage of the distal joint surface of proximal phalanx is thoroughly curetted (D), the skin flaps are pulled over the stump and closed using simple interrupted suture pattern by silk $(E)$, removal of suture 15 days after complete wound healing (F).

Table 2.Sedation score was documented by a score system modified after Aithal et al., (1996).

\begin{tabular}{|c|c|c|}
\hline $\begin{array}{l}\text { Sedation } \\
\text { score }\end{array}$ & Clinical sedation & Description of sedation \\
\hline 0 & No sedation & The animal appeared alert \\
\hline 1 & Mild sedation & $\begin{array}{l}\text { Slight drowsiness, but animal } \\
\text { response to auditory stimuli }\end{array}$ \\
\hline 2 & $\begin{array}{l}\text { Moderate } \\
\text { sedation }\end{array}$ & $\begin{array}{l}\text { Dropping head, eyelid closed } \\
\text { swaying of the body }\end{array}$ \\
\hline 3 & Severe sedation & The animal unable to stand \\
\hline
\end{tabular}

Table 3. Lameness score definitions according to Anzuino et al., (2010).

\begin{tabular}{ll} 
Score & \multicolumn{1}{c}{ Definition } \\
\hline 0 & $\begin{array}{l}\text { Goat places full weight on all four limbs, moves } \\
\text { forward freely with an even gait }\end{array}$ \\
1 & $\begin{array}{l}\text { Goat has a definite limp on one or more legs, but } \\
\text { bearing weight and moves forward freely }\end{array}$ \\
& $\begin{array}{l}\text { Goat has some difficulty moving forward, severe } \\
\text { limp, bearing little weight on one or more legs, may } \\
\text { be a degree of goose-stepping }\end{array}$ \\
& $\begin{array}{l}\text { Goat has some difficulty moving forward, non- } \\
\text { weight bearing on one or more legs, or may 'goose- } \\
\text { step' high or walk on the knees }\end{array}$
\end{tabular}




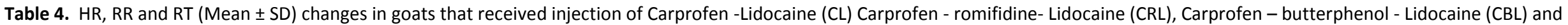
Carprofen, romifidine, butterphenol, Lidocaine (CRBL) groups .

\begin{tabular}{|c|c|c|c|c|c|c|c|c|c|c|c|}
\hline \multirow[b]{2}{*}{$\begin{array}{l}\text { Variable } \\
\text { s }\end{array}$} & \multicolumn{5}{|c|}{ Surgery } & \multicolumn{6}{|c|}{ Post-surgery } \\
\hline & groups & Base line & $\begin{array}{l}\text { 15min After } \\
\text { anesthesia }\end{array}$ & $\begin{array}{l}\text { Start of } \\
\text { surgery }\end{array}$ & $\begin{array}{l}\text { End of } \\
\text { surgery }\end{array}$ & $30 \mathrm{~min}$ & $60 \mathrm{~min}$ & $120 \mathrm{~min}$ & 1 day & 2 day & 3 day \\
\hline \multirow{4}{*}{$\begin{array}{l}\text { HR } \\
\text { (beat / } \\
\text { min) }\end{array}$} & $\mathrm{L}$ & $106.25 \pm 13.70^{a}$ & $105 \pm 12.90^{\mathrm{a}}$ & $115.5 \pm 10.87^{\mathrm{a}}$ & $138 \pm 4.89^{* a}$ & $123.2 \pm 5.37^{* a}$ & $104.25 \pm 3.30^{\mathrm{a}}$ & $112.5 \pm 6.45^{\mathrm{a}}$ & $119.75 \pm 3.86^{\mathrm{a}}$ & $122.75 \pm 1.5^{\mathrm{a}}$ & $122.50 \pm 4.20^{\mathrm{a}}$ \\
\hline & $\mathrm{CL}$ & $119 \pm 1.15^{\mathrm{a}}$ & $135.5 \pm 5.74^{b}$ & $128 \pm 9.52^{\mathrm{a}}$ & $121 \pm 3.82^{\mathrm{a}}$ & $129 \pm 2.94^{\mathrm{a}}$ & $128.75 \pm 5.5^{\mathrm{b}}$ & $123 \pm 6.78^{\mathrm{a}}$ & $138.25 \pm 5.3^{b}$ & $96.50 \pm 6.7^{* b}$ & $101.50 \pm 5.7^{b}$ \\
\hline & CRL & $122 \pm 2.82^{\mathrm{a}}$ & $87 \pm 5.29^{* c}$ & $79.7 \pm 3.30^{*^{b}}$ & $73.75 \pm 3.5^{*^{b}}$ & $105.25 \pm 5.2^{b}$ & $108.25 \pm 7.67^{\mathrm{a}}$ & $118.25 \pm 9.17^{\mathrm{a}}$ & $125.75 \pm 5.67^{c}$ & $127.25 \pm 3.59^{\mathrm{a}}$ & $113.75 \pm 4.34^{\mathrm{a}}$ \\
\hline & CRBL & $114 \pm 5.16^{\mathrm{a}}$ & $70.7 \pm 4.57^{* c}$ & $63.2 \pm 4.27^{*^{\mathrm{b}}}$ & $57.5 \pm 2.21^{* b}$ & $61.25 \pm 3.9^{* c}$ & $71.50 \pm 3^{* c}$ & $76.25 \pm 2.1^{* \mathrm{~b}}$ & $104.7 \pm 13.5^{d}$ & $122.75 \pm 8.38^{\mathrm{a}}$ & $111.75 \pm 7.22^{\mathrm{a}}$ \\
\hline \multirow{4}{*}{$\begin{array}{l}\text { RR } \\
\text { (breath/ } \\
\text { min) }\end{array}$} & $\mathrm{L}$ & $20 \pm 1.63^{\mathrm{a}}$ & $18 \pm 2.82^{\mathrm{a}}$ & $16.25 \pm 2.06^{\mathrm{a}}$ & $16.25 \pm 0.95^{\mathrm{a}}$ & $16.75 \pm 0.5^{\mathrm{a}}$ & $15.25 \pm 1.89^{\mathrm{a}}$ & $17.25 \pm 1.5^{\mathrm{a}}$ & $19.75 \pm 0.95^{\mathrm{a}}$ & $19.5 \pm 1^{\mathrm{a}}$ & $21.75 \pm 2.87^{\mathrm{ab}}$ \\
\hline & $\mathrm{CL}$ & $21.75 \pm 1.7^{\mathrm{a}}$ & $18.25 \pm 0.95^{\mathrm{a}}$ & $20 \pm 0.00^{\mathrm{a}}$ & $18.25 \pm 0.5^{\mathrm{a}}$ & $21.25 \pm 0.95^{\mathrm{b}}$ & $16.75 \pm 1.7^{\mathrm{ab}}$ & $18.75 \pm 0.95^{\mathrm{a}}$ & $21.5 \pm 1^{\mathrm{ab}}$ & $20.75 \pm 0.95^{\mathrm{a}}$ & $18.75 \pm 1.7^{\mathrm{a}}$ \\
\hline & CRL & $19.5 \pm 0.75^{\mathrm{a}}$ & $17.75 \pm 0.95^{\mathrm{a}}$ & $16.5 \pm 1.29^{\mathrm{a}}$ & $18.25 \pm 3.77^{\mathrm{a}}$ & $16.5 \pm 2.38^{\mathrm{a}}$ & $17.75 \pm 3.9^{\mathrm{ab}}$ & $18.25 \pm 0.5^{\mathrm{a}}$ & $20.75 \pm 0.95^{\mathrm{ab}}$ & $19.25 \pm 1.7^{\mathrm{a}}$ & $21.75 \pm 0.5^{\mathrm{ab}}$ \\
\hline & CRBL & $23.75 \pm 1.7^{\mathrm{a}}$ & $19.5 \pm 2.08^{\mathrm{a}}$ & $19.5 \pm 1^{a}$ & $17 \pm 1.41^{* a}$ & $17.75 \pm 1.2^{\mathrm{ab} *}$ & $19 \pm 0.00^{b}$ & $20.5 \pm 1^{\mathrm{a}}$ & $23 \pm 1.82^{b}$ & $26.5 \pm 3.09^{b}$ & $24.5 \pm 1^{b}$ \\
\hline \multirow{4}{*}{$\begin{array}{l}\text { RT } \\
\text { (oc) }\end{array}$} & $\mathrm{L}$ & $39.37 \pm 0.33^{\mathrm{a}}$ & $39.35 \pm 0.01^{\mathrm{a}}$ & $39.35 \pm 0.05^{\mathrm{a}}$ & $39.55 \pm 0.12^{\mathrm{a}}$ & $39.25 \pm 0.12^{\mathrm{a}}$ & $39.17 \pm 0.09^{\mathrm{a}}$ & $39.17 \pm 0.12^{\mathrm{a}}$ & $38.8 \pm 0.08^{\mathrm{a}}$ & $38.85 \pm 0.12^{\mathrm{a}}$ & $39.15 \pm 0.12^{\mathrm{a}}$ \\
\hline & $\mathrm{CL}$ & $39.12 \pm 0.01^{\mathrm{a}}$ & $39.22 \pm 0.67^{\mathrm{a}}$ & $39.37 \pm 0.51^{\mathrm{a}}$ & $39.25 \pm 0.3^{\mathrm{a}}$ & $39 \pm 0.18^{\mathrm{a}}$ & $38.95 \pm 0.19^{\mathrm{a}}$ & $37.95 \pm 1.07^{b}$ & $38.85 \pm 0.4^{\mathrm{a}}$ & $38.92 \pm 0.51^{\mathrm{a}}$ & $39.05 \pm 0.23^{\mathrm{a}}$ \\
\hline & CRL & $39.1 \pm 0.08^{\mathrm{a}}$ & $38.57 \pm 0.63^{\mathrm{a}}$ & $39.52 \pm 0.45^{\mathrm{ab}}$ & $39.47 \pm 0.35^{\mathrm{a}}$ & $39.12 \pm 0.26^{\mathrm{a}}$ & $39.17 \pm 0.27^{\mathrm{a}}$ & $38.47 \pm 1.06^{c}$ & $39.32 \pm 0.65^{\mathrm{a}}$ & $39.2 \pm 0.47^{\mathrm{ab}}$ & $39.1 \pm 0.14^{\mathrm{a}}$ \\
\hline & CRBL & $39.17 \pm 0.09^{\mathrm{a}}$ & $40.05 \pm 0.05^{b}$ & $39.97 \pm 0.12^{b}$ & $39.67 \pm 0.09^{a}$ & $39.1 \pm 0.08^{\mathrm{a}}$ & $39.15 \pm 0.12^{\mathrm{a}}$ & $38.7 \pm 0.23^{c d}$ & $38.75 \pm 0.23^{\mathrm{a}}$ & $39.62 \pm 0.09^{b}$ & $39.25 \pm 0.12^{\mathrm{a}}$ \\
\hline
\end{tabular}

Corresponding means with different superscripts differ significantly $(P<0.05)$ among groups.

Means with an asterisk $(*)$ differ significantly $(P<0.05)$ from baseline. 
Table 5. Behavioral alterations (Mean \pm SD) in goats that received injection of Carprofen -Lidocaine (CL), Carprofen romifidine- Lidocaine (CRL), Carprofen - buterphenol Lidocaine (CBL) and Carprofen, romifidine, buterphenol, Lidocaine (CRBL) groups

\begin{tabular}{|c|c|c|c|c|c|c|c|}
\hline \multicolumn{4}{|c|}{ Surgery } & \multicolumn{4}{|c|}{ Post-surgery } \\
\hline Variables & Groups & During & End & $15 \mathrm{~min}$ & $30 \mathrm{~min}$ & $1 \mathrm{~h}$ & $2 \mathrm{~h}$ \\
\hline \multirow[t]{4}{*}{ Struggling (n / $10 \mathrm{~min}$ ) } & L & $2.2 \pm .0^{\mathrm{a}}$ & $1.5 \pm .0^{\mathrm{a}}$ & $2.0 \pm .0^{\mathrm{a}}$ & $2.0 \pm .0^{\mathrm{a}}$ & $2.0 \pm 0.0^{\mathrm{a}}$ & $2.0 \pm 0.0^{\mathrm{a}}$ \\
\hline & $\mathrm{CL}$ & $0.0 \pm 0.0^{c}$ & $.55 \pm .33^{\mathrm{b}}$ & $1.0 \pm .44^{\mathrm{ab}}$ & $1.3 \pm .6^{\mathrm{ab}}$ & $2.0 \pm 0.0^{\mathrm{a}}$ & $2.0 \pm 0.0^{\mathrm{a}}$ \\
\hline & CRL & $.8 \pm .3^{\mathrm{b}}$ & $.4 \pm .3^{\mathrm{b}}$ & $.0 \pm .0^{\mathrm{b}}$ & $.3 \pm .3^{b}$ & $1.3 \pm .3^{\mathrm{a}}$ & $2.0 \pm 0.0^{\mathrm{a}}$ \\
\hline & CRBL & $0.0 \pm 0.0^{c}$ & $.67 \pm .3^{b}$ & $.0 \pm .3^{\mathrm{b}}$ & $0.0 \pm 0.0^{b}$ & $.3 \pm .3^{\mathrm{b}}$ & $1.3 \pm .66^{\mathrm{a}}$ \\
\hline \multirow[t]{4}{*}{ Vocalization (n / $10 \mathrm{~min}$ ) } & $\mathrm{L}$ & $2.0 \pm .0^{\mathrm{a}}$ & $2.0 \pm .0^{\mathrm{a}}$ & $2.0 \pm .0^{\mathrm{a}}$ & $2.0 \pm .0^{\mathrm{a}}$ & $2.0 \pm 0.0^{\mathrm{a}}$ & $2.0 \pm 0.0^{\mathrm{a}}$ \\
\hline & $\mathrm{CL}$ & $.33 \pm .33^{b}$ & $.66 \pm .33^{b}$ & $.66 \pm .6^{\mathrm{ab}}$ & $.66 \pm .6^{b}$ & $2.0 \pm 0.0^{\mathrm{a}}$ & $2.0 \pm 0.0^{b}$ \\
\hline & CRL & $.33 \pm .33^{b}$ & $.66 \pm .33^{\mathrm{b}}$ & $.33 \pm .33^{\mathrm{ab}}$ & $.0 \pm .0^{c}$ & $.66 \pm .3^{\mathrm{b}}$ & $1.3 \pm .6^{\mathrm{ab}}$ \\
\hline & CRBL & $.0 \pm .33^{b}$ & $.33 \pm .33^{\mathrm{b}}$ & $.0 \pm .0^{\mathrm{b}}$ & $.0 \pm .0^{c}$ & $.33 \pm .33^{b}$ & $1.3 \pm .6^{\text {ab }}$ \\
\hline \multirow[t]{4}{*}{ Head shaking ( $\mathrm{n} / 10 \mathrm{~min}$ ) } & $\mathrm{L}$ & $2.0 \pm .0^{\mathrm{a}}$ & $2.0 \pm .0^{\mathrm{a}}$ & $2.0 \pm .0^{\mathrm{a}}$ & $2.0 \pm .0^{\mathrm{a}}$ & $2.0 \pm .0^{\mathrm{a}}$ & $0.0 \pm 0.0^{\mathrm{a}}$ \\
\hline & $\mathrm{CL}$ & $0.0 \pm 0.0^{b}$ & $0.0 \pm 0.0^{b}$ & $0.0 \pm 0.0^{b}$ & $0.0 \pm 0.0^{b}$ & $0.0 \pm 0.0^{b}$ & $0.0 \pm 0.0^{\mathrm{a}}$ \\
\hline & CRL & $0.0 \pm 0.0^{b}$ & $0.0 \pm 0.0^{b}$ & $0.0 \pm 0.0^{b}$ & $0.0 \pm 0.0^{b}$ & $0.0 \pm 0.0^{b}$ & $0.0 \pm 0.0^{\mathrm{a}}$ \\
\hline & CRBL & $0.0 \pm 0.0^{b}$ & $0.0 \pm 0.0^{b}$ & $0.0 \pm 0.0^{\mathrm{b}}$ & $0.0 \pm 0.0^{b}$ & $0.0 \pm 0.0^{b}$ & $0.0 \pm 0.0^{\mathrm{a}}$ \\
\hline \multirow[t]{4}{*}{ Ear flicking ( $\mathrm{n} / 10 \mathrm{~min}$ ) } & $\mathrm{L}$ & $2.0 \pm .0^{\mathrm{a}}$ & $2.0 \pm .0^{\mathrm{a}}$ & $2.0 \pm .0^{\mathrm{a}}$ & $2.0 \pm .0^{\mathrm{a}}$ & $2.0 \pm .0^{a}$ & $0.0 \pm 0.0^{\mathrm{a}}$ \\
\hline & $\mathrm{CL}$ & $0.0 \pm 0.0^{b}$ & $0.0 \pm 0.0^{b}$ & $0.0 \pm 0.0^{b}$ & $0.0 \pm 0.0^{b}$ & $0.0 \pm 0.0^{b}$ & $0.0 \pm 0.0^{\mathrm{a}}$ \\
\hline & CRL & $0.0 \pm 0.0^{b}$ & $0.0 \pm 0.0^{b}$ & $0.0 \pm 0.0^{b}$ & $0.0 \pm 0.0^{b}$ & $0.0 \pm 0.0^{b}$ & $0.0 \pm 0.0^{\mathrm{a}}$ \\
\hline & CRBL & $0.0 \pm 0.0^{b}$ & $0.0 \pm 0.0^{b}$ & $0.0 \pm 0.0^{b}$ & $0.0 \pm 0.0^{b}$ & $0.0 \pm 0.0^{b}$ & $0.0 \pm 0.0^{\mathrm{a}}$ \\
\hline
\end{tabular}

Corresponding means with different superscripts differ significantly $(P<0.05)$ among groups.

Table 6. Sedation score (Mean \pm SD) in goats that received injection of Carprofen -Lidocaine (CL), Carprofen r- romifidine- Lidocaine (CRL), Carprofen - buterphenol - Lidocaine $(\mathrm{CBL})$ and Carprofen, romifidine, buterphenol, Lidocaine (CRBL) groups.

\begin{tabular}{|c|c|c|c|c|c|c|c|c|}
\hline Variables & groups & $\begin{array}{l}\text { Base } \\
\text { Line }\end{array}$ & $\begin{array}{l}\text { After inject } \\
\text { of anesthetic }\end{array}$ & $\begin{array}{l}\text { Start of } \\
\text { surgery }\end{array}$ & $\begin{array}{l}\text { End of } \\
\text { surgery }\end{array}$ & $\begin{array}{l}30 \text { min post- } \\
\text { surgery }\end{array}$ & $\begin{array}{l}60 \text { min post- } \\
\text { surgery }\end{array}$ & $\begin{array}{l}120 \mathrm{~min} \\
\text { post -surgery }\end{array}$ \\
\hline \multirow[t]{4}{*}{ Sedation score } & L & $0.00 \pm 0.00$ & $1.00 \pm 0.00^{\mathrm{a}}$ & $1.00 \pm 0.00^{\mathrm{a}}$ & $1.00 \pm 0.00^{\mathrm{a}}$ & $1.00 \pm 0.00^{\mathrm{a}}$ & $1.00 \pm 0.00^{\mathrm{a}}$ & $1.00 \pm 0.00^{\mathrm{a}}$ \\
\hline & $\mathrm{CL}$ & $0.00 \pm 0.00$ & $1.00 \pm 0.00^{\mathrm{a}}$ & $1.00 \pm 0.00^{\mathrm{a}}$ & $1.00 \pm 0.00^{\mathrm{a}}$ & $1.00 \pm 0.00^{\mathrm{a}}$ & $1.00 \pm 0.00^{\mathrm{a}}$ & $1.00 \pm 0.00^{\mathrm{a}}$ \\
\hline & $\mathrm{CRL}$ & $0.00 \pm 0.00$ & $1.75 \pm 0.5^{\mathrm{b}}$ & $2.25 \pm 0.5^{b}$ & $2.25 \pm 0.57^{b}$ & $2.00 \pm 0.00^{b}$ & $2.25 \pm 0.50^{b}$ & $1.75 \pm 0.50^{b}$ \\
\hline & CRBL & $0.00 \pm 0.00$ & $2.25 \pm 0.5^{b}$ & $2.75 \pm 0.5^{b}$ & $2.75 \pm 0.5^{b}$ & $2.75 \pm 0.5^{b}$ & $3.00 \pm 0.00^{b}$ & $2.75 \pm 0.50^{\mathrm{b}}$ \\
\hline
\end{tabular}

Corresponding means with different superscripts differ significantly $(\mathrm{P}<0.05)$ among groups. 
Table 7. lameness score (Mean \pm SD) in goats that received injection of Carprofen -Lidocaine (CL), Carprofen r- romifidine- Lidocaine (CRL), Carprofen - buterphenol Lidocaine (CBL) and Carprofen, romifidine, buterphenol, Lidocaine (CRBL) groups .

\begin{tabular}{|c|c|c|c|c|c|c|c|c|c|}
\hline Variables & groups & Baseline & $15 \mathrm{~min} P O$ & $30 \mathrm{~min} P O$ & $60 \mathrm{~min} P O$ & $120 \mathrm{~min} P O$ & 1 day PO & 2 day $P O$ & 3day PO \\
\hline \multirow{4}{*}{$\begin{array}{l}\text { Lameness } \\
\text { score }\end{array}$} & $\mathrm{L}$ & $0.00 \pm 0.00$ & $3.00 \pm 0.81$ & $3.25 \pm 0.95^{\mathrm{a}}$ & $2.75 \pm 0.50^{\mathrm{a}}$ & $3.00 \pm 0.81^{\mathrm{a}}$ & $.3 .50 \pm 0.57^{\mathrm{a}}$ & $3.00 \pm 0.81^{\mathrm{a}}$ & $3.75 \pm 0.95^{\mathrm{a}}$ \\
\hline & $\mathrm{CL}$ & $0.00 \pm 0.00$ & $2.75 \pm 0.50$ & $3.00 \pm 0.81^{\mathrm{a}}$ & $2.50 \pm 0.57^{\mathrm{a}}$ & $2.50 \pm 0.57^{\mathrm{a}}$ & $2.25 \pm 0.50^{\mathrm{a}}$ & $2.25 \pm 0.50^{\mathrm{a}}$ & $2.00 \pm 0.81^{\mathrm{a}}$ \\
\hline & CRL & $0.00 \pm 0.00$ & $2.25 \pm 0.50$ & $3.00 \pm 0.81^{a}$ & $2.00 \pm 0.00^{b}$ & $1.75 \pm 0.50^{b}$ & $1.50 \pm 0.57^{b}$ & $1.50 \pm 0.57^{b}$ & $1.50 \pm 0.57^{b}$ \\
\hline & CRBL & $0.00 \pm 0.00$ & $3.00 \pm 0.81$ & $2.50 \pm 1.00^{b}$ & $2.00 \pm 0.81^{b}$ & $1.50 \pm 0.57^{b}$ & $1.50 \pm 0.57^{b}$ & $2.00 \pm 0.00^{\mathrm{a}}$ & $1.50 \pm 0.57^{b}$ \\
\hline
\end{tabular}

Corresponding means with different superscripts differ significantly $(P<0.05)$ among groups.

Table 8. Interleukin- 6 changes (Mean \pm SD) in goats that received injection of Carprofen -Lidocaine (CL), Carprofen romifidine- Lidocaine (CRL), Carprofen buterphenol - Lidocaine (CBL) and Carprofen, romifidine, buterphenol, Lidocaine (CRBL) groups.

\begin{tabular}{|c|c|c|c|c|c|c|c|c|}
\hline Variables & groups & Baseline & During surgery & 60 min PO & 120 min $P O$ & 1 day $P O$ & 2 day $P O$ & 3 day $P O$ \\
\hline \multirow{4}{*}{ IL- $6 \quad(p g / m l)$} & $\mathrm{L}$ & $3.57 \pm 0.49^{\mathrm{a}}$ & $7.26 \pm 0.57^{* a}$ & $8.71 \pm 1.33^{* a}$ & $7.1 \pm 1.53^{* a}$ & $6.26 \pm 1.31^{\mathrm{a}}$ & $5.54 \pm 0.76^{\mathrm{a}}$ & $4.34 \pm 0.56^{\mathrm{a}}$ \\
\hline & $\mathrm{CL}$ & $5.64 \pm 0.51^{\mathrm{a}}$ & $19.8 \pm 2.48^{* \mathrm{~b}}$ & $15.22 \pm 2.67^{* b}$ & $9.97 \pm 2.69^{* \mathrm{~b}}$ & $5.81 \pm 0.57^{\mathrm{a}}$ & $4.88 \pm 0.82^{\mathrm{a}}$ & $3.7 \pm 0.97^{\mathrm{a}}$ \\
\hline & CRL & $6.20 \pm 1.01^{\mathrm{a}}$ & $5.18 \pm 2.25^{\mathrm{c}}$ & $6.44 \pm 0.34^{\mathrm{a}}$ & $6.03 \pm 0.24^{\mathrm{c}}$ & $6.26 \pm 0.12^{\mathrm{a}}$ & $5.77 \pm 0.85^{\mathrm{a}}$ & $5.89 \pm 0.93^{\mathrm{a}}$ \\
\hline & CRBL & $5.67 \pm 0.60^{\mathrm{a}}$ & $3.6 \pm 3.73^{* d}$ & $5.05 \pm 0.73^{\mathrm{a}}$ & $5.55 \pm 0.87^{\mathrm{dc}}$ & $7.73 \pm 1.17^{\mathrm{a}}$ & $8.11 \pm 2.44^{* b}$ & $14.29 \pm 7.9^{* \mathrm{~b}}$ \\
\hline
\end{tabular}

Corresponding means with different superscripts differ significantly $(P<0.05)$ among groups. Means with an asterisk $(*)$ differ significantly $(P<0.05)$ from baseline.

Table 9. Serum lactate level in goats that received injection of Carprofen -Lidocaine (CL), Carprofen r- romifidine- Lidocaine (CRL), Carprofen - butterphenol Lidocaine (CBL) Carprofen, romifidine, butterphenol, Lidocaine (CRBL) groups (Mean $\pm S D$ ).

\begin{tabular}{|c|c|c|c|c|c|c|c|c|c|c|}
\hline $\begin{array}{l}\text { Varia } \\
\text { ble }\end{array}$ & groups & $\mathrm{BL}$ & During & End & $30 \mathrm{~min}$ & $60 \mathrm{~min}$ & $120 \mathrm{~min}$ & 1 day & 2day & 3 day \\
\hline \multirow{4}{*}{$\begin{array}{l}\text { Lactate } \\
\text { (mg/dl) }\end{array}$} & L & $25.62 \pm 0.47^{\mathrm{a}}$ & $32.5 \pm 0.77^{\mathrm{a}}$ & $57.9 \pm 24.5^{*^{\mathrm{a}}}$ & $50.95 \pm 8.4^{* a}$ & $45.52 \pm 19.2^{\mathrm{a}}$ & $48.2 \pm 6.24^{\mathrm{a}}$ & $50.5 \pm 13.3^{*^{a}}$ & $37.1 \pm 2.68^{\mathrm{a}}$ & $35.35 \pm 22.4^{\mathrm{a}}$ \\
\hline & $\mathrm{CL}$ & $26.32 \pm 0.47^{\mathrm{a}}$ & $31.32 \pm 3.55^{\mathrm{a}}$ & $36.87 \pm 2.05^{b}$ & $44.1 \pm 0.45^{* b}$ & $28.4 \pm 0.4^{b}$ & $38.4 \pm 6.26^{b}$ & $57.37 \pm 4.9^{* \mathrm{~b}}$ & $37.85 \pm 0.3^{\mathrm{a}}$ & $48.82 \pm 6.23^{*^{b}}$ \\
\hline & CRL & $79.6 \pm 10.92^{b}$ & $50.4 \pm 11.67^{b}$ & $31.6 \pm 6.6^{* b}$ & $43.4 \pm 1.73^{*^{b}}$ & $40.1 \pm 11.3^{*^{\mathrm{a}}}$ & $35.22 \pm 5.8^{*^{b}}$ & $49.85 \pm 7.8^{\mathrm{a}}$ & $32.47 \pm 3.6^{*^{b}}$ & $39.6 \pm 19.48^{*^{a}}$ \\
\hline & CRBL & $53.15 \pm 1.38^{c}$ & $43.7 \pm 16.79^{c}$ & $32.72 \pm 4.89 *^{\mathrm{b}}$ & $43.4 \pm 1.32^{b}$ & $31.9 \pm 4.74^{* b}$ & $32.8 \pm 0.81 *^{\mathrm{b}}$ & $49.67 \pm 4.63^{\mathrm{a}}$ & $34.5 \pm 3.99 *^{2 \mathrm{ab}}$ & $34.52 \pm 10.57^{*^{a}}$ \\
\hline
\end{tabular}

Corresponding means with different superscripts differ significantly $(P<0.05)$ among groups. Means with an asterisk $(*)$ differ significantly $(P<0.05)$ from baseline. 


\section{DISCUSSION}

Lameness is substantial welfare issue in small ruminant [9]. In sheep, lameness has been associated with weight loss[1], decreased fertility and lamb growth rates[1].In contrast, very little research has been undertaken into lameness in dairy goats. Previous observations on dairy goat farms estimated the prevalence of lameness to be between 9.1 and $19.2 \%[1,3]$. In sheep, $90 \%$ of lameness cases are caused by foot rot (Dichelobacter nodosus) [3]. D. nodosus has also been confirmed as a cause of lameness in goats[4]. However,[20] found only one of four dairy goat farms investigated to be affected by foot rot. The objective of this study was to evaluate the effect of IVRA in a multimodal analgesic protocol in goats undergoing digit amputation through cardiopulmonary, biochemical, metabolic, behavioral alteration, sedation score and lameness score for alleviation of pain in goats undergoing unilateral claw amputation.

In this study romifidine treated animals showed significantly reduced $H R$ and $R R$ as well as excellent analgesia. Additionally, IL-6 remained significantly lower in romifidine treated animals compared to other groups. The use of romfidine in the analgesic protocol during surgical intervention in cases of digit amputation in goats can alleviates surgical stress and potentiates the analgesic effects of IVRA[14, 21].

HR in IVRA treated animals showed a non-significant increase except at the end of surgery and $30 \mathrm{~min}$ PO. this result could be attributed to the sympathetic nerve block and vasodilatation induced by the lidocaine[22, 23]. IVRA has a rapid onset to perform and more reliable compared to nerve block and is therefore the method of choice for surgery of bovine digit[14] for such reason in the present study IVRA was used for anesthesia and analgesia of distal limb in goats undergoing unilateral digit amputation.

Pre-treatment with carprofen ameliorate the physiological responses to surgical intervention, the result of present study suggest that carprofen may provide some analgesia to extensive claw surgery in goats this results agreed with [11] who suggest that carprofen may provide some analgesia to traumatic procedure in sheep

The mean $\mathrm{HR}$ of $\mathrm{CL}$ treated animals showed significant increased. This result could be attributed to the sympathetic nerve block and vasodilatation induced by the lidocaine [22, 23]. In this study, there was an initial increase of HR followed by gradual decrease in $\mathrm{CL}$ treated animals.

The results of HR were significantly decreased in CRBL treated animals. This reduction in HR may be resulted from cardiovascular and respiratory depressants effect of butorphenol [24]. Than CLR and this was concise with HR significantly increased following administration of butorphenol in goats by relaxing vascular musculature[25].

A significantly decreased in $H R$ was shown after administration of romfidine may be due to the effect of alphaagonist which induced bradycardia which included a decrease in sympathetic outflow from the CNS, inhibition of norepinephrine release from sympathetic nerve terminals, direct depression of cardiac pacemaker and conduction tissue, increased vagal tone and a direct increase in the release of acetylcholine from parasympathetic nerves in heart[26, 27] .

Intravenous injection of romifidine showed a significant reduction in $R R$ at the end of surgery due to respiratory depression associated with alpha-2 agonists might be secondary to the CNS depression produced by alpha- 2 adrenoceptor stimulation[27] or to the result of direct depression of the respiratory centers by pre-anaesthetics [25].

A significant decrease in RT was recorded in CRL treated animals at 120 p.o, this might be attributed to a decrease in the skeletal muscle tone, reduced metabolic rates and muscle relaxation, along with depression of thermoregulatory centers [28]. Alpha-2 agonists have been reported to induce prolonged depression of thermoregulation[29]. Hypothermia tends to be the most common response, particularly when opioids, lidocaine and midazolam are used [24, 30] .

Interleukin-6 is secreted by a wide range of cells including immune cells, fibroblasts, endothelial cells, and neurons. It is one of the most common cytokines that induces the hyperalgesia[31]. Preemptive IVRA and $C L$ treatment has significant increase of IL- 6 effect to attenuate IL-6. These results agreed with[22] who found that lidocaine does not abolish the pain associated with surgery and the strong stimulation of nociceptors during the surgical treatment leads to a direct release of cytokines into the bloodstream which subsequently triggers an endocrine stress response. [32] added that, IL6 causes production of small mediators (cyclooxygenase 2) which generates PGE2 that causes vasodilatation and enhances perception of pain. In the present study, addition of carprofen had a significant attenuation of serum IL-6 during and post-surgery in CL, CLR and CLRB groups. This may be due to decrease of inflammatory cells production that responsible for IL-6 secretion after surgical incision[31, 32].

The preemptive combination of romifidine-carprofenbuterphenol results in highest attenuation of serum IL-6 compared to the other groups. Romifidine was able to increase the ability of carprofen to inhibit prostaglandin synthesis via the blockage of cyclooxygenase and the prevention of cytokines induced hyperalgesia [33]. This result agreed with the finding of [34] who reported that stimulation of serotonergic descending inhibitory system resulted in 
decreased stimulation of lymphocyte proliferation and decrease of IL-6 production and buterphenol increase time of sedation and analgesia.

In the present study, lactate level increased significantly in IVRA, CL, but tended to be higher in lidocaine treated animals. This could be attributed to stress induced by surgical intervention. A mild increase in blood lactate concentrations were reported in cattle during abdominal surgeries and were attributed to stress induced vasoconstriction and reduced tissue oxygenation[35, 36]. [35]added that, the increase in serum lactate activity may be attributed to possible muscle damage. Plasma lactate remained on average lower in bucks of CLR, CLRB, group than in IVRA groups and CL, This result agreed with[14] who reported that, plasma lactate remained on average lower in cows of the xylazine group than in controls and agreed with [17] in goats undergoing tube cystotomy.

After injection of romifidine in CRL treated animals there was a significant moderate sedative and analgesic effect this result agreed with [37] . They reported that romifidine has been used for sedation of horses, dogs, sheep and goats. In CRBL treated animals there was severe sedation and analgesia at $30 \mathrm{~min}$ p.o. and increase time of sedation compared with CRL, this agreed with[38] who mentioned that opioid and alpha 2- agonist agent are synergistic in terms of sedation and analgesia. Sedation in this study clearly show that goats more sensitive to alpha 2 agonist (romifidine) than in horses the drugs are usually used in doses that enable the animals to remain standing although with marked ataxia, the combination of romifidine and buterphnol in the fourth group caused longer standing, sedation and ataxia in goats [38].

There was a significant increase in lameness score in IVRA treated animals p.o. surgery from $30 \mathrm{~min}$ till third day. There was moderate lame in $\mathrm{CL}$ treated animals p.o. surgery from 30 min till third day. There was moderate lame till $60 \mathrm{~min}$ p.o surgery followed by mild lame till third day in CRL treated animals. In CRBL treated animals there was a moderate lameness till 60 min p.o surgery followed by mild lame till third day p.o. surgery. There was a significant decreased in degree of lame in CRL and CRBL compared with first and second group. Lameness is an expression of pain in goats subjected to claw surgery and can be semi quantitatively assessed by lameness score[9]. In carprofen treated cows, a quick reduction in lameness score can be expected after digital amputation when inflammatory affected tissue is almost completely removed.

In this study the lameness score improved after surgery careprofen treated goats. Reduction of lameness score after NSAIDs treatment have seen demonstrated in previous studies in lame cattle[39, 40].
Due to a synergism analgesic effects of local anaesthetics are prolonged when used in combination with alph2-agonist in sheep undergoing orthopaedic surgery[16]. As in other studies [16] changes in cow's behaviour such as lameness scoring, appetite and changes on pain induced behaviour changes were used to evaluate the stress and pain perceived by goats during claw amputation after romifidine pre-treatment. However, the use of behavioural parameters to assess pain and to determine the analgesic effectiveness of drugs presents a challenging task[10, 14]. In comparison to controls romifidine-buterphanol pre-treated goats exhibited in this study significantly less frequent ear flicking during the surgical intervention at the claws, lower lameness scores and longer standing periods during the first hour after claw treatment, and more frequently immediate start of feed consumption when goat arrived back in their individual free stall after claw surgery.

All these behaviour alterations may indicate less pain perception and improved well-being of goats. No group differences were found for other behaviours such as head shaking, tail wagging and vocalisation. We conclude therefore that the pre-treatment with romifidine in combination with IVRA improved and prolonged to some extent analgesia compared to IVRA alone.

There is a limitation in this study; we need to expand our research to study the effect of the used anesthetic and analgesic combinations in clinical cases with extensive claw horn lesion in goats.

\section{Conclusion}

It was concluded that, carprofen analgesic and inflammatory properties may be used to complement local anesthesia (IVRA) during and after surgical intervention on the goat digit post -surgery. And its combination with buterphanol provides excellent analgesia for digit amputation in goats.

Romifidine appears to be and appropriate sedative for pain treatment in goats undergoing claw amputation and can be used as analgesic in a multimodal protocol for short term pain management during claw surgery in goats.

Further work is also required on the impact of other NSAID in combination with sedative and analgesics in goats clinically affected with extensive claw horn lesion.

\section{Conflict of interest statement}

The authors declare that there is no any conflict of interest in the current research work

\section{Animal ethics committee permission}

The current research work is permitted to be executed according to standards of animal Research committee in Faculty of Veterinary Medicine, Mansoura University. 


\section{Authors contributions:}

Usama fekry and Awad Rizk performed the experiment, statistical analysis, research writing, Esam Mosbah and Adel Zaghloul : revised manuscript and supervise the whole work

\section{REFERENCES}

[1] Anzuino K, Bell N, Bazeley K, Nicol C. Assessment of welfare on 24 commercial UK dairy goat farms based on direct observations. Vet Rec 2010;167:774-80.

[2] Sibley R. Lameness in dairy cows: the developing story. Vet. Rec 2013;172:92-5.

[3] Winter AC. Treatment and control of hoof disorders in sheep and goats. Vet Clin: Food Animal Pract 2011;27:187-92.

[4] Zhou H, Bennett G, Hickford JG. Variation in Fusobacterium necrophorum strains present on the hooves of footrot infected sheep, goats and cattle. Vet Microbiol 2009;135:363-7.

[5] Sullivan L, Clegg S, Angell J, Newbrook K, Blowey R, Carter S, et al. Highlevel association of bovine digital dermatitis Treponema spp. with contagious ovine digital dermatitis lesions and presence of Fusobacterium necrophorum and Dichelobacter nodosus. J of Clini Microbiol 2015;53:1628-38.

[6] Duran SP, Manzano JV, Valera RC, Machota SV. Susceptibilities of Bacteroides and Fusobacterium spp. from foot rot in goats to 10 betalactam antibiotics. Antimicrob Agents Chemother 1990;34:657-9.

[7] Christodoulopoulos G. Foot lameness in dairy goats. Res Vet Sci 2009;86:281-4.

[8] Mgasa M, Arnbjerg J. Radiographic study of postnatal development of the tarsus in west African dwarf goats. Anat Histol Embryol 1993;22:16-25.

[9] Groenevelt M, Anzuino K, Smith S, Lee MR, Grogono-Thomas R. A case report of lameness in two dairy goat herds; a suspected combination of nutritional factors concurrent with treponeme infection. BMC Res Notes 2015;8:791.

[10] Valverde A, Gunkel Cl. Pain management in horses and farm animals. J Vet Emer and Crit Car 2005;15:295-307.

[11] Paull D, Lee C, Colditz I, Atkinson S, Fisher A. The effect of a topical anaesthetic formulation, systemic flunixin and carprofen, singly or in combination, on cortisol and behavioural responses of Merino lambs to mulesing. Aust Vet J 2007;85:98-106.

[12] Colditz I, Lloyd J, Paull D, Lee C, Giraudo A, Pizzato C, et al. Effect of the non-steroidal anti-inflammatory drug, carprofen, on weaned sheep following non-surgical mulesing by intradermal injection of cetrimide. Aust Vet J 2009;87:19-26.

13] Heppelmann M, Kofler J, Meyer H, Rehage J, Starke A. Advances in surgical treatment of septic arthritis of the distal interphalangeal joint in cattle: A review. Vet J 2009;182:162-75.

[14] Rizk A, Herdtweck S, Offinger J, Meyer H, Zaghloul A, Rehage J. The use of xylazine hydrochloride in an analgesic protocol for claw treatment of lame dairy cows in lateral recumbency on a surgical tipping table. Vet J 2012;192:193-8.

[15] Babalola G, Oke B. Intravenous regional analgesia for surgery of the limbs in goats. Vet Quart 1983;5:186-9.

16] Graf B, Senn M. Behavioural and physiological responses of calves to dehorning by heat cauterization with or without local anaesthesia. Appl Anim Behav Sci 1999;62:153-71.

[17] Hamed M, Rizk A, Mosbah E, Abonorg M, Karrouf G. Evaluation of Different Anesthetic Drugs Combination for Pain Management in Goats Undergoing Tube Cystostomy. G Vet 2015; 14 (2): 251-261.

[18] Aithal H, Pratap AK, Singh G. Clinical effects of epidurally administered ketamine and xylazine in goats. Small Ruminant Res 1997;24:55-64.

[19] Kofler J, Fessl L. Comparison of the methods of amputation through phalanx media versus exarticulation in the proximal interphalangeal joint in cattle.Wien TierarztMonat 1989;76:224-30.

[20] Hill N, Murphy P, Nelson A, Mouttotou N, Green L, Morgan K. Lameness and foot lesions in adult British dairy goats. Vet Rec 1997;141:412-6.
[21] Freeman S, Bowen I, Bettschart-Wolfensberger R, Alibhai H, England G. Cardiovascular effects of romifidine in the standing horse. Res in Vet Sci 2002;72:123-9.

[22] Doherty T, Kattesh H, Adcock R, Welborn M, Saxton A, Morrow J, et al. Effects of a concentrated lidocaine solution on the acute phase stress response to dehorning in dairy calves. J DS 2007; 90:4232-9.

[23] Habibian S, Bigham A, Aali E. Comparison of lidocaine, tramadol, and lidocaine-tramadol for epidural analgesia in lambs. Res Vet Sci 2011;91:434-8

[24] DeRossi R, Almeida RG, Medeiros $U$, Righetto FR, Frazílio FO. Subarachnoid butorphanol augments lidocaine sensory anaesthesia in calves. Vet J 2007;173:658-63.

[25] Carroll GL, Hooper RN, Slater MR, Hartsfield SM, Matthews NS Detomidine-butorphanol-propofol for carotid artery translocation and castration or ovariectomy in goats. Vet Surg1998;27:75-82.

[26] MacDonald E, Virtanen R. Review of pharmacology of medetomidine and detomidine: two chemically similar alpha-2-adrenoreceptor agonists used as veterinary sedatives. Ani pain1992; 181-191

[27] Sinclair MD. A review of the physiological effects of $\alpha 2$-agonists related to the clinical use of medetomidine in small animal practice. CanVet 2003;44:885.

[28] Ponder S, Clark W. Prolonged depression of thermoregulation after xylazine administration to cats. JVPT 1980; 3:203-7.

[29] Kästner SB, Boller J, Kutter AP, Pakarinen SM, Ramela MP, Huhtinen MK Comparison of cardiopulmonary effects of dexmedetomidine administered as a constant rate infusion without loading dose in sheep and goats anaesthetised with sevoflurane. Small Ruminant Res 2007;71:75-82.

[30] Dzikiti TB, Stegmann GF, Dzikiti LN, Hellebrekers LJ. Effects of midazolam on isoflurane minimum alveolar concentration in goats. Small Ruminant Res 2011;97:104-9.

[31] Rincon M. Interleukin-6: from an inflammatory marker to a target for inflammatory diseases. Trends Immunol 2012;33:571-7.

[32] Rose-John S, Scheller J, Elson G, Jones SA. Interleukin-6 biology is coordinated by membrane-bound and soluble receptors: role in inflammation and cancer. J Leuko Bio 2006;80:227-36.

[33] Ingvast-Larsson C, Högberg M, Mengistu U, Olsen L, Bondesson U, Olsson K. Pharmacokinetics of meloxicam in adult goats and its analgesic effect in disbudded kids.JVPT 2011;34:64-9.

[34] Fransson BA, Lagerstedt AS, Bergstrom A, Hagman R, Park JS, Chew BP, et al. C-reactive protein, tumor necrosis factor $\alpha$, and interleukin- 6 in dogs with pyometra and SIRS. JVECC 2007;17:373-81.

[35] El-Ghoul W, Hofmann W. Influence of claw diseases of varying degrees on measurable stress reactions with special consideration of cortisol and lactate in bovine blood serum. P Tier-Hannover 2002;83:354-61.

[36] Mudroň P, Rehage J, Sallmann H, Höltershinken M, Scholz H. Stress Response in Dairy Cows Related to Different Blood Glucose. Acta Vet Brno 2005; 74:37-42.

[37] Celly C, McDonell W, Young S, Black W. The comparative hypoxaemic effect of four $\alpha 2$ adrenoceptor agonists (xylazine, romifidine, detomidine and medetomidine) in sheep. JVPT 1997; 20:464-71.

[38] El-Kammar M, Gad S, Korittum A. Evaluation of the Sedative, Analgesic, Physiological and Haematological Effects of Intravenous Detomidine, Detomidine-Butorphanol, Romifidine and Romifidine-Butorphanol in Baladi Goats. G Vet 2014;12:36-44

[39] Whay H, Webster A, Waterman-Pearson A. Role of ketoprofen in the modulation of hyperalgesia associated with lameness in dairy cattle. Vet Rec 2005;157:729-33.

[40] Offinger J, Herdtweck S, Rizk A, Starke A, Heppelmann M, Meyer H, et al. Postoperative analgesic efficacy of meloxicam in lame dairy cows undergoing resection of the distal interphalangeal joint. JDJ 2013:96:866-76. 